\title{
MULTIPLICITIES AND LOG CANONICAL THRESHOLD
}

TOMMASO DE FERNEX, LAWRENCE EIN, AND MIRCEA MUSTAŢǍ

\section{INTRODUCTION}

Let $X$ be a smooth algebraic variety, defined over an algebraically closed field, of characteristic zero. Consider a closed subscheme $Y \subset X$, and let $Z$ be an irreducible component of $Y$, of codimension $n$. We study the connection between two of the basic invariants of the ideal $I$ defining $Y$ in $\mathcal{O}_{X, Z}$.

The first invariant is very classical: it is the Samuel multiplicity $e(I)$ of $\mathcal{O}_{X, Z}$ along $I$. The second invariant has been much studied recently, due to its proeminent role in higher dimensional birational geometry. It is called the log canonical threshold of $\mathcal{O}_{X, Z}$ along $I$, and it is denoted by $\operatorname{lc}(I)$.

Our main result is the following:

Theorem 0.1. With the above notation, we have

$$
e(I) \geq \frac{n^{n}}{\operatorname{lc}(I)^{n}}
$$

The case $n=2$ of the above theorem was proved by Corti in Co. We also characterize the case when in (11) we have equality: this happens if and only if the integral closure of $I$ is a power of the maximal ideal in $\mathcal{O}_{X, Z}$.

If $Z \subseteq Y$, but it is not an irreducible component of $Y$, then $I$ is not zero-dimensional, so $e(I)$ is not defined. In this case, Gaffney and Gassler have introduced in [GG the Segre numbers of $\mathcal{O}_{X, Z}$ along $I$, denoted by $e_{i}(I)$, for $i=1, \ldots, n$. One can formulate a possible generalization of Theorem 0.1 to this setting (see Question 1.5 below). We prove this in the case $n=2$. More precisely, we show the following:

1991 Mathematics Subject Classification. Primary 14B05; Secondary 14C17.

Key words and phrases. Multiplicity, log canonical threshold, monomial ideals.

Research of the second author was partially supported by NSF Grant DMS 9970295. The third author served as a Clay Mathematics Institute Long-Term Prize Fellow while this research has been done. 
Theorem 0.2. If $Z \subset X$ is a closed and irreducible subset of codimension 2, contained in the support of $Y$, and if $e_{1}(I)$ and $e_{2}(I)$ are the Segre numbers of $\mathcal{O}_{X, Z}$ along $I$, then

$$
\frac{4}{\operatorname{lc}(I)} e_{1}(I)+e_{2}(I) \geq \frac{4}{\operatorname{lc}(I)^{2}} .
$$

In fact, in the two-dimensional case, we give also a stronger version of this inequality (see Theorem 2.4 below).

The proofs of these results are based on deformation to monomial ideals. To fix the ideas, we discuss now the case of Theorem 0.1. We prove first an inequality between the length and the log canonical threshold:

$$
l(R / I)>\frac{n^{n}}{n ! \cdot \operatorname{lc}(I)^{n}} .
$$

The inequality in Theorem 0.1 follows by applying (3) to the powers $I^{t}$, dividing by $t^{n}$, and passing to the limit.

In order to prove (3), standard arguments allow us to reduce the general case to that of an ideal $J$ in a polynomial ring. By considering a deformation to a monomial ideal in $(J)$, it is enough to prove (3) when $J$ is monomial. We achieve this via a combinatorial argument.

Interest in bounds for log canonical thresholds is partly motivated by applications to birational rigidity. Iskovskikh and Manin proved in [IM that a smooth quartic in $\mathbb{P}^{4}$ is birationally rigid (hence, in particular, it is not rational), and this has started a whole direction in higher dimensional birational geometry (see, for example [Pu2], [Pu3], [CPR ). Recently, Corti initiated in [CD a new approach to this circle of ideas, based on Shokurov's Connectedness principle and the twodimensional case of inequality (1). In this spirit, we apply Theorem 0.1 in [DEM] to give an upper bound for the log canonical threshold of a generic projection of a projective variety. This improves a bound due to Pukhlikov from [Pu1], bound which plays a major role in his proof of the fact that a smooth hypersurface of degree $n$ in $\mathbb{P}^{n}, n \geq 4$, is birationally rigid.

0.1. Acknowledgements. We are grateful to Rob Lazarsfeld for many useful conversations.

\section{LOWER-BOUNDS TO LENGTH AND MULTIPLICITY}

Throughout this paper, all varieties are assumed to be defined over an algebraically closed field $k$ of characteristic zero. We first fix the notation. 
Let $X$ be a smooth variety, $Y \subset X$ a closed subscheme, and let $Z$ be an irreducible component of $Y$. We denote by $I \subset \mathcal{O}_{X, Z}$ the ideal locally defining $Y$. Let $\operatorname{lc}(I)$ be the log canonical threshold of $\left(\mathcal{O}_{X, Z}, I\right)$, and set $\mu(I)=1 / \operatorname{lc}(I)$. For the definition and basic properties of the log canonical threshold, we refer to [Ko]. Note that following the definition in $[\mathrm{Kg}], \operatorname{lc}(I)$ is the maximum of $\operatorname{lc}(U, U \cap Y)$, when $U \subseteq X$ is an open subset such that $U \cap Z \neq \emptyset$.

Let $\operatorname{codim}_{X} Z=n$. We denote by $l\left(\mathcal{O}_{X, Z} / I\right)$ the length of $\mathcal{O}_{X, Z} / I$, and by $e(I)$ the multiplicity of $\mathcal{O}_{X, Z}$ along $I$. Our main result is the following theorem.

Theorem 1.1. With the above notation, we have

$$
l\left(\mathcal{O}_{X, Z} / I\right)>\frac{n^{n} \mu(I)^{n}}{n !} .
$$

The above result easily gives the theorem we have stated in the Introduction.

Theorem 1.2. Keeping the above notation, we have

$$
e(I) \geq n^{n} \mu(I)^{n} \text {. }
$$

Proof. By applying Theorem 1.1 to powers $I^{t}$ of $I$, we get

$$
l\left(\mathcal{O}_{X, Z} / I^{t}\right) \geq \frac{n^{n} \mu\left(I^{t}\right)^{n}}{n !} .
$$

where $\mu\left(I^{t}\right)$ is the inverse of the log canonical threshold of $\left(\mathcal{O}_{X, Z}, I^{t}\right)$. Since

$$
\mu\left(I^{t}\right)=t \mu(I) \quad \text { and } \quad \lim _{t} \frac{n ! \cdot l\left(\mathcal{O}_{X, Z} / I^{t}\right)}{t^{n}}=e(I),
$$

(5) follows by multiplying both sides of (6) by $n ! / t^{n}$ and passing to the limit as $t \rightarrow \infty$.

The proof of Theorem 1.1 consists of a reduction to the case of monomial ideals, when the assertion follows from the explicit description of the log canonical threshold. Before proving Theorem 1.1, we recall the definition and the main properties of the Newton polytope associated to a monomial ideal. To a monomial $x_{1}^{a_{1}} \cdots x_{n}^{a_{n}}$ we associate the point $\left(a_{1}, \ldots, a_{n}\right) \in \mathbb{N}^{n} \subset \mathbb{R}_{+}^{n}$ (here $\mathbb{R}_{+}=[0,+\infty)$ ). If $J \subset R=K\left[x_{1}, \ldots, x_{n}\right]$ is a monomial ideal, the Newton polytope $P(J)$ associated to $J$ is defined as the convex hull in $\mathbb{R}_{+}^{n}$ of the points corresponding to the monomials in $J$. Let $\operatorname{lc}(J)$ be the log canonical threshold of $(R, J)$, and set $\mu(J)=1 / \operatorname{lc}(J)$. Then a result due to Howald [H] implies that

$$
\mu(J)=\min \{\alpha>0 \mid \alpha \cdot \mathbf{e} \in P(J)\},
$$


where $\mathbf{e}=(1, \ldots, 1) \in \mathbb{R}_{+}^{n}$. We will also write $\mu(P(J))$ for $\mu(J)$. Moreover, we extend this notation, defining

$$
\mu(P)=\inf \{\alpha>0 \mid \alpha \cdot \mathbf{e} \in P\},
$$

for any subset $P \subset \mathbb{R}_{+}^{n}$, such that $\alpha \cdot \mathbf{e} \in P$, for some $\alpha>0$.

We observe that $J$ is a zero dimensional ideal if and only if $\mathbb{R}_{+}^{n} \backslash$ $P(J)$ is bounded in $\mathbb{R}_{+}^{n}$. We define the volume of a zero dimensional monomial ideal $J$ by setting

$$
\operatorname{Vol}(J):=n ! \operatorname{Vol}\left(\mathbb{R}_{+}^{n} \backslash P(J)\right),
$$

where the right hand side is the volume of the corresponding bounded region, in the Euclidean metric. 巴

Lemma 1.3. If $J \subset R$ is a zero dimensional monomial ideal, then $n ! \cdot l(R / J)>\operatorname{Vol}(J)$.

Proof. We denote by $\left(u_{1}, \ldots, u_{n}\right)$ the coordinates on $\mathbb{R}^{n}$. Let

$$
C_{0}=\left\{\left(u_{1}, \ldots, u_{n}\right) \in \mathbb{R}_{+}^{n} \mid 0 \leq u_{i} \leq 1\right\}
$$

be the standard unitary $n$-cube of $\mathbb{R}_{+}^{n}$. For any point $a=\left(a_{1}, \ldots, a_{n}\right) \in$ $\left(\mathbb{N}^{n} \backslash P(J)\right)$, let $\tau_{a}: \mathbb{R}_{+}^{n} \rightarrow \mathbb{R}_{+}^{n}$ be the translation which sends the origin to the point $a$, and consider the $n$-cube $C_{a}=\tau_{a}\left(C_{0}\right)$. Then

$$
\begin{aligned}
l(R / J) & =\#\{\text { monomials } \notin J\} \\
& \geq \#\left(\mathbb{N}^{n} \backslash P(J)\right)=\operatorname{Vol}\left(\bigcup_{a \in\left(\mathbb{N}^{n} \backslash P(J)\right)} C_{a}\right) .
\end{aligned}
$$

Note that a point $u=\left(u_{1}, \ldots, u_{n}\right) \in\left(\mathbb{R}_{+}^{n} \backslash P(J)\right)$ is contained in the $n$-cube $C_{[u]}$, where $[u]=\left(\left[u_{1}\right], \ldots,\left[u_{n}\right]\right) \in\left(\mathbb{N}^{n} \backslash P(J)\right)([]$ denotes the integral part operator). Therefore the union of the $n$-cubes appearing on the right hand side covers $\mathbb{R}^{n} \backslash P(J)$. This proves the weak inequality in the statement. To prove that the inequality is strict, it is enough to note that $\mathbb{R}_{+}^{n} \backslash \bigcup_{a \in\left(\mathbb{N}^{n} \backslash P(J)\right)} C_{a}$ is not convex, hence $\bigcup_{a \in\left(\mathbb{N}^{n} \backslash P(J)\right)} C_{a} \cap$ $P(J)$ has positive volume.

Proof of Theorem 1.1. By passing to completion, we obtain a zero dimensional ideal $\widehat{I} \subset \widehat{\mathcal{O}}_{X, Z}$. Note that $\widehat{\mathcal{O}}_{X, Z}$ is isomorphic to a formal power series ring $K\left[\left[x_{1}, \ldots, x_{n}\right]\right]$. We will identify these two $k$-algebras under some fixed isomorphism. Then, since $\widehat{I}$ is zero dimensional, we can find an ideal $J$ in the polynomial ring $R=K\left[x_{1}, \ldots, x_{n}\right]$, with $\operatorname{Supp}(V(J))=\{0\} \subset \mathbb{A}^{n}$, and whose completion in $K\left[\left[x_{1}, \ldots, x_{n}\right]\right]$ is $\widehat{I}$.

\footnotetext{
${ }^{1}$ For a zero dimensional monomial ideal $J, \operatorname{Vol}(J)=e(J)$, but we will not need this fact.
} 
Since we have an isomorphism of $k$-algebras $\mathcal{O}_{X, Z} \simeq R / J$, it is clear that $l\left(\mathcal{O}_{X, Z} / I\right)=l(R / J)$. Moreover, we have $\operatorname{lc}\left(\mathcal{O}_{X, Z}, I\right)=\operatorname{lc}(R, J)$. To see this, recall that if $W \subset \widetilde{W}$ is a subscheme of a smooth variety $\widetilde{W}$ over some field $F$, then $\operatorname{dim}(\widetilde{W})-\operatorname{lc}(\widetilde{W}, W)$ depends only on $W$, but not on the embedding $W \subset \widetilde{W}$ (see, for example, [Mu], Corollary 3.5). Therefore it is enough to note that computing $\operatorname{lc}\left(\mathbb{A}_{K}^{n}, V(J)\right)$ (over $K$ ) is the same as computing $\operatorname{lc}\left(\mathbb{A}_{k}^{n} \times T, V(J)\right)$ (over $k$ ), where $T$ is a suitable smooth variety over $k$, with function field $K$.

The above argument shows that we may reduce ourselves to the case of the ideal $J \subset R$. By an extension of scalars to the algebraic closure of $K$, we may also assume that $K$ is algebraically closed. The proof now splits into two parts. First we prove the theorem assuming that $J$ is a monomial ideal. Then we deduce the inequality for arbitrary $J$ by a degeneration argument.

Suppose that $J$ is a monomial ideal. Let $F$ be the facet of $P(J)$ containing the point $\mu \cdot \mathbf{e}$. Since $F$ is bounded (recall that $J$ is zero dimensional), the equation of the hyperplane supporting $F$ can be written as

$$
\sum_{i=1}^{n} u_{i} / a_{i}=1
$$

for some $a_{i}>0$. This gives

$$
\operatorname{Vol}(J) \geq \prod_{i=1}^{n} a_{i} \quad \text { and } \quad \mu(J)=\left(\sum_{i=1}^{n} \frac{1}{a_{i}}\right)^{-1} .
$$

Then, by comparing the arithmetic and geometric means of $\left\{1 / a_{i}\right\}_{i}$, we see that

$$
\operatorname{Vol}(J) \geq n^{n} \mu(J)^{n} .
$$

Therefore the theorem follows, in the monomial case, by Lemma 1.3 .

Now we consider an arbitrary ideal $J \subset R=K\left[x_{1}, \ldots, x_{n}\right]$ with $\operatorname{Supp}(R / J)=\{0\} \subset \mathbb{A}^{n}$. After fixing a multiplicative order on the coordinates $x_{i}$, we can take a deformation to the initial monomial ideal (see [Ei], Chapter 15). This is a flat family $\left\{J_{s}\right\}_{s \in K}$ such that $S / J_{s} \cong$ $S / J$, for all $s \neq 0$, and such that $\operatorname{in}(J):=J_{0}$ is a monomial ideal. Since this is a flat deformation, $\operatorname{Supp}(R / \operatorname{in}(J))=\{0\}$ and $l(R / J)=$ $l(R /$ in $(J))$. Moreover, the semicontinuity property of the log canonical threshold (see, for example, DK]) gives $\operatorname{lc}(R, J) \geq \operatorname{lc}(R, \operatorname{in}(J)$ ), hence $\mu(J) \leq \mu(\operatorname{in}(J))$. Thus inequality (5) follows now from the case of monomial ideals.

The boundary case in Theorem 1.2 is characterized in the following theorem. 
Theorem 1.4. Under the assumptions of Theorem 1.9,

$$
e(I)=n^{n} \mu(I)^{n}
$$

if and only if there is a positive integer $q$, such that the integral closure $\bar{I}$ of $I$ is equal to $\mathcal{M}^{q}$, where $\mathcal{M}$ is the maximal ideal of $\mathcal{O}_{X, Z}$. Moreover, in this case $q=n \mu(I)$.

Proof. If we assume that $\bar{I}=\mathcal{N}^{q}$ for some positive integer $q$, then $e(I)=q^{n}$ and $\operatorname{lc}(I)=n / q$, hence (77) is satisfied.

Conversely, assume that (7) holds. Since $e(I) \in \mathbb{N}$ and $\mu(I) \in \mathbb{Q}_{+}$, we see that $n \mu(I) \in \mathbb{N}$. We will prove that

$$
\bar{I}=\mathcal{M}^{n \mu(I)} \text {. }
$$

By hypothesis, $e(I)=e\left(\mathcal{M}^{n \mu(I)}\right)$, and we will show that

$$
I \subseteq \mathcal{M}^{n \mu(I)}
$$

This will imply our assertion by a theorem of Rees [R], which says that if two zero dimensional ideals $I \subseteq J \subset \mathcal{O}_{X, Z}$ are such that $e(I)=e(J)$, then $\bar{I}=\bar{J}$.

Therefore it is enough to prove (8). Arguing as in the beginning of the proof of Theorem 1.1, we reduce to study the case when $I$ is an ideal in $R=K\left[x_{1}, \ldots, x_{n}\right]$ with $\operatorname{Supp}(R / I)=\{0\} \subset \mathbb{A}^{n}$.

We deform all the powers $I^{t}$ to monomial ideals in $\left(I^{t}\right)$, as follows. Consider a fixed multiplicative order on the monomials in $R$. We first deform $I^{t}$ to the tangent cone, i.e., to the ideal generated by the sum of terms of lowest degree in $f$, for $f \in I^{t}$, and then take the initial ideal of the resulting ideal, with respect to the monomial order. By the way we have made the deformation, $I \subseteq \mathcal{M}^{p}$ if and only if in $(I) \subseteq \mathcal{M N}^{p}$. Note also that in $\left(I^{t}\right) \cdot \operatorname{in}\left(I^{s}\right) \subseteq \operatorname{in}\left(I^{s+t}\right)$.

As in the proof of Theorem 1.1, for every positive integer $t$,

$$
\frac{n ! \cdot l\left(R / \operatorname{in}\left(I^{t}\right)\right)}{t^{n}} \geq \frac{n^{n} \mu\left(\operatorname{in}\left(I^{t}\right)\right)^{n}}{t^{n}} \geq \frac{n^{n} \mu\left(I^{t}\right)^{n}}{t^{n}}=n^{n} \mu(I)^{n} .
$$

On the other hand, since we are assuming that $e(I)=n^{n} \mu(I)^{n}$, we also have

$$
\frac{n ! \cdot l\left(R / \operatorname{in}\left(I^{t}\right)\right)}{t^{n}}=\frac{n ! \cdot l\left(R / I^{t}\right)}{t^{n}} \rightarrow e(I)=n^{n} \mu(I)^{n},
$$

for $t \rightarrow \infty$. Combining these two formulas, we obtain

$$
\lim _{t \rightarrow \infty} \frac{\mu\left(\operatorname{in}\left(I^{t}\right)\right)}{t}=\mu(I) .
$$

Set

$$
P_{t}=\frac{1}{t} P\left(\operatorname{in}\left(I^{t}\right)\right) \quad \text { and } \quad P_{\infty}=\cup_{r \in \mathbb{N}} P_{2^{r}} .
$$


Note that for every pair of positive integers $r \leq s, P_{2^{r}} \subseteq P_{2^{s}}$. This follows from the fact that

$$
\left(\operatorname{in}\left(I^{2^{r}}\right)\right)^{2^{s-r}} \subseteq \operatorname{in}\left(I^{2^{s}}\right) .
$$

Therefore $P_{\infty}$ is a convex subset in $\mathbb{R}_{+}^{n}$, and

$$
\mu\left(P_{\infty}\right)=\lim _{r \rightarrow \infty} \frac{\mu\left(\operatorname{in}\left(I^{2^{r}}\right)\right)}{2^{r}}=\mu(I) .
$$

On the other hand, an application of Lemma 1.3 gives

$$
\begin{aligned}
n ! \operatorname{Vol}\left(\mathbb{R}_{+}^{n} \backslash P_{\infty}\right) & =\lim _{r \rightarrow \infty}\left(n ! \operatorname{Vol}\left(\mathbb{R}_{+}^{n} \backslash P_{2^{r}}\right)\right) \\
& \leq \lim _{r \rightarrow \infty} \frac{n ! \cdot l\left(R / \operatorname{in}\left(I^{2^{r}}\right)\right)}{\left(2^{r}\right)^{n}}=n^{n} \mu(I)^{n} .
\end{aligned}
$$

By the convexity of $P_{\infty}$, we can find an hyperplane $F \subset \mathbb{R}_{+}^{n}$ passing through the point $\mu\left(P_{\infty}\right) \cdot \mathbf{e}$ and disjoint from the interior of $P_{\infty}$. Let $\sum_{i=1}^{n} u_{i} / a_{i}=1$ be the equation of $F$, and denote by $S_{F}$ the simplex in $\mathbb{R}_{+}^{n}$ containing the origin and having $F$ as diagonal facet. Then we have

$$
n^{n} \mu(I)^{n} \leq n ! \operatorname{Vol}\left(S_{F}\right) \leq n ! \operatorname{Vol}\left(\mathbb{R}_{+}^{n} \backslash P_{\infty}\right)=n^{n} \mu(I)^{n} .
$$

Thus this is a chain of equalities. This implies the equality between the arithmetic and geometric means of $\left\{1 / a_{i}\right\}_{i}$, which can happen only if all $a_{i}$ are equal. Therefore we conclude that $a_{i}=n \mu(I)$ for all $i$. Observing that $P(\operatorname{in}(I))=P_{1} \subseteq P_{\infty}$, we deduce that in $(I) \subseteq \mathcal{M}^{n \mu(I)}$. Therefore $I \subseteq \mathcal{M}^{n \mu(I)}$, which concludes the proof of the theorem.

In the remaining part of this section, we discuss a more general set up. As before, we consider a closed subscheme $Y$ of a smooth algebraic variety $X$ together with an irreducible subvariety $Z$ of $X$. However, now we only assume that $Z$ is contained in $Y$. In other words, the ideal $I \subset \mathcal{O}_{X, Z}$, locally defining $Y$, is not necessarily zero dimensional. We would like to generalize inequality (5) in this setting.

Segre numbers, introduced by Gaffney and Gassler in [GG], appear as a natural choice for a substitute for the Samuel multiplicity in this more general context. Like the Samuel multiplicity of a zero dimensional ideal, the Segre numbers of the ideal $I$ can be computed as intersection numbers and have a natural interpretation as multiplicities, related to the Vogel cycle associated to $I$. The following seems a plausible generalization of Theorem 1.2. 
Question 1.5. With the above notation, if $n=\operatorname{codim}_{X} Z$, and if $e_{1}(I), \ldots, e_{n}(I)$ are the Segre numbers of $I$, is it true that

$$
\sum_{k=1}^{n} \frac{e_{k}(I)}{k^{k} \mu(I)^{k}} \geq 1 \text { ? }
$$

Note that if $I \subset \mathcal{O}_{X, Z}$ is a complete intersection ideal, of dimension $r$, then $e_{i}(I)=0$ for $i \neq n-r$, and $e_{n-r}(I)=e\left(I+\left(f_{1}, \ldots, f_{r}\right)\right)$, where $f_{1}, \ldots, f_{r}$ are general linear combinations of a system of generators of I. In particular, the inequality (9) holds, by applying Theorem 1.4 for the ring $\mathcal{O}_{X, Z} /\left(f_{1}, \ldots, f_{r}\right)$. This also shows that the coefficients before the Segre numbers in (9) are optimal.

In the next section, we will give a positive answer to the above question in the codimension two case.

\section{INEQUALITIES IN CODIMENSION TWO}

In this section we concentrate on the codimension two case. Specifically, we show that the inequality in Question 1.5 holds in this case. We provide also a sharper inequality when the ambient variety is a surface. First we prove the following lemma.

Lemma 2.1. Let $I$ be a monomial ideal in the ring $R=K\left[x_{1}, x_{2}\right]$, and let $\mu(I)$ denote the inverse of the log canonical threshold $\operatorname{lc}(I)$ of $(R, I)$. Write $I=x_{1}^{b_{1}} x_{2}^{b_{2}} \cdot \mathfrak{a}$, where $\mathfrak{a}$ is a zero dimensional ideal. Then the length of $R / \mathfrak{a}$ is bounded by:

$$
l(R / \mathfrak{a}) \geq 2\left(\mu(I)-b_{1}\right)\left(\mu(I)-b_{2}\right) .
$$

Proof. Note first that

$$
\mu(I) \geq \max \left\{b_{1}, b_{2}\right\} .
$$

If we have equality, then (10) is trivially satisfied, so from now on we assume that this is not the case.

Let $P(I)$ and $P(\mathfrak{a})$ be the Newton polytopes associated to $I$ and $\mathfrak{a}$, respectively, and let

$$
U=\left\{\left(u_{1}, u_{2}\right) \in \mathbb{R}_{+}^{2} \mid u_{i} \geq b_{i}\right\} .
$$

If $\tau_{b}$ is the translation sending the origin of $\mathbb{R}_{+}^{2}$ to the point $b=\left(b_{1}, b_{2}\right)$, we have

$$
\tau_{b}\left(\mathbb{R}_{+}^{2} \backslash P(\mathfrak{a})\right)=U \backslash P(I) .
$$

The boundary of $P(I)$ is supported on the union of finitely many lines, among which the two lines $l_{1}: u_{1}=b_{1}$ and $l_{2}: u_{2}=b_{2}$. Let $l$ be the line supporting a facet of $P(I)$ which contains the point $(\mu(I), \mu(I))$. Note that $l$ is neither $l_{1}$ nor $l_{2}$, since we have assumed $\mu(I)>\max \left\{b_{1}, b_{2}\right\}$. 
Let $T \subset \mathbb{R}_{+}^{2}$ be the triangular region bounded by the three lines $l, l_{1}, l_{2}$. Because of the convexity of $P(I)$, we see that $T \subseteq U \backslash P(I)$. Then we have

$$
\operatorname{Vol}(\mathfrak{a}) \geq 2 \operatorname{Vol}(T)
$$

hence, by Lemma 1.3,

$$
l(R / \mathfrak{a}) \geq \operatorname{Vol}(T) .
$$

Let $S \subset \mathbb{R}_{+}^{2}$ be the square with vertices $(0,0),(\mu(I), 0),(0, \mu(I))$, and $(\mu(I), \mu(I)) . S \cap U$ is a rectangle of area $\left(\mu(I)-b_{1}\right)\left(\mu(I)-b_{2}\right)$, and

$$
S \cap U \subset T \text {. }
$$

Then inequality (10) follows by observing that the area of a rectangle inscribed in a right triangle (with the edges of the rectangle parallel to the legs of the triangle) does not exceed half of the area of the triangle. This is an easy consequence of the inequality between the arithmetic and the geometric means. (Alternatively, one can give a synthetic proof of this fact by simply drawing a picture and suitably doubling the rectangle.)

Before applying Lemma 2.1, we fix some notation. Let $X$ be a smooth variety, $Z \subset X$ an irreducible subvariety of codimension 2, and $Y \subset X$ a subscheme containing $Z$. Let $I \subset \mathcal{O}_{X, Z}$ be the ideal locally defining $Y$. We can write $I=f \cdot \mathfrak{a}$, where $f \in \mathcal{O}_{X, Z}$ and $\mathfrak{a}$ is zero dimensional. We denote by $\mu(I)$ the inverse of the log canonical threshold of $(R, I)$, by mult $Z(f)$ the multiplicity of $f$ at the generic point of $Z$ and by $e(\mathfrak{a})$ the Samuel multiplicity of $\mathfrak{a}$. We have the following theorem.

Theorem 2.2. With the above notation, we have

$$
4 \mu(I) \operatorname{mult}_{Z}(f)+e(\mathfrak{a}) \geq 4 \mu(I)^{2} .
$$

Proof. Note that it is enough to prove that the following inequality holds:

$$
4 \mu(I) \operatorname{mult}_{Z}(f)+2 l\left(\mathcal{O}_{X, Z} / \mathfrak{a}\right) \geq 4 \mu(I)^{2} .
$$

Indeed, since $I^{t}=f^{t} \cdot \mathfrak{a}^{t}$ for every $t \in \mathbb{N}$, we can apply (12) to powers $I^{t}$ of $I$, so that inequality (11) follows by dividing both sides by $t^{2}$ and passing to the limit as $t \rightarrow \infty$, as in the proof of Theorem 1.2.

The idea is to deform $I$ to a monomial ideal, and to deduce the inequality (12) from Lemma 2.1. If the log canonical center of $\left(\mathcal{O}_{X, Z}, \operatorname{lc}(I) \cdot I\right)$ has codimension one, then we are done, as in this case $\mu(I) \leq$ mult $_{Z}(f)$. Therefore we may assume that this is not the case.

We proceed as in the proof of Theorem 1.1. Passing to completion, we obtain an ideal $\widehat{I}$ in $\widehat{\mathcal{O}}_{X, Z}$. We identify $\widehat{\mathcal{O}}_{X, Z}$ with $K\left[\left[x_{1}, x_{2}\right]\right]$ via 
a fixed isomorphism. This gives a decomposition $\widehat{I}=g \cdot \mathfrak{b}$, with $\mathfrak{b}$ a zero dimensional ideal. Since $\mathfrak{b}$ is zero dimensional, there is an ideal $\mathbf{c} \subset R=K\left[x_{1}, x_{2}\right]$ such that $\mathfrak{b}=\widehat{\mathbf{c}}$.

We fix now a monomial order on $R$ and then consider $\operatorname{in}(g)$ and $\operatorname{in}(\mathbf{c})$. Here $\operatorname{in}(g)=x_{1}^{b_{1}} x_{2}^{b_{2}}$ is the largest of the monomials in $g$ of smallest degree, and in(c) is the initial ideal, under the fixed order, of the ideal obtained from $\mathbf{c}$ after deforming to the normal cone. We put $J=x_{1}^{b_{1}} x_{2}^{b_{2}} \cdot \operatorname{in}(\mathbf{c})$. As before, we have

$$
\operatorname{lc}(I)=\operatorname{lc}(g \cdot \mathbf{c}) \geq \operatorname{lc}(J),
$$

and $l\left(\mathcal{O}_{X, Z} / I\right)=l\left(R / x_{1}^{b_{1}} x_{2}^{b_{2}} \cdot \operatorname{in}(\mathbf{c})\right)$. Note also that $\operatorname{mult}_{Z}(f)=b_{1}+b_{2}$.

Lemma 2.1, applied to $J$, implies

$$
l(R / \text { in }(\mathbf{c})) \geq 2 \mu(J)^{2}-2 \mu(J)\left(b_{1}+b_{2}\right) .
$$

If we put $\alpha=\mu(J)-\mu(I)$, then $\alpha \geq 0$, and

$$
l\left(\mathcal{O}_{X, Z} / \mathfrak{a}\right) \geq 2(\mu(I)+\alpha)^{2}-2(\mu(I)+\alpha) \operatorname{mult}_{Z}(f) .
$$

Then, by expanding the right hand side and noting that $\mu(I) \geq$ $\frac{1}{2} \operatorname{mult}_{Z}(f)$, we get

$$
l\left(\mathcal{O}_{X, Z} / \mathfrak{a}\right) \geq 2 \mu(I)^{2}-2 \mu(I) \operatorname{mult}_{Z}(f),
$$

hence the inequality (12).

We show now that Theorem 2.2 gives a positive answer to Question 1.5 in the codimension two case.

Theorem 2.3. Under the hypothesis of Theorem 2.9, if $e_{1}(I), e_{2}(I)$ are the Segre numbers of $I$, then

$$
4 \mu(I) e_{1}(I)+e_{2}(I) \geq 4 \mu(I)^{2} .
$$

Proof. Let $W:=\operatorname{Spec} \mathcal{O}_{X, Z}$, and recall that we denote by $\mathcal{M}$ the closed point of $W$. If $I$ is a principal ideal, then the result is trivial. Henceforth, we will assume that $I$ is not principal. We recall now how the Segre numbers are defined in the two dimensional case (see GG] for the general case). Consider the fiber product diagram

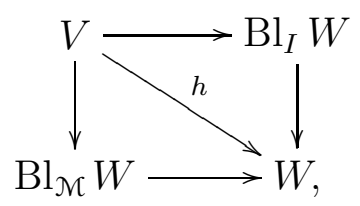

where $\mathrm{Bl}_{I} W$ and $\mathrm{Bl}_{\mathcal{M}} W$ are the blow-ups along $I$ and $\mathcal{M}$, respectively. We can write

$$
I \cdot \mathcal{O}_{V}=\mathcal{O}_{V}\left(-E_{I}\right) \text { and } \mathcal{M} \cdot \mathcal{O}_{V}=\mathcal{O}_{V}\left(-E_{\mathcal{M}}\right)
$$


for some effective Cartier divisors $E_{I}, E_{\mathcal{M}}$ on $V$. We denote by $E_{I}^{Z}$ the union of the components of $E_{I}$ which are mapped to $\mathcal{M}$, and set $E_{I}^{X \backslash Z}=E_{I}-E_{I}^{Z}$. The Segre numbers of $I$ are then given by

$$
e_{1}(I)=E_{I}^{X \backslash Z} \cdot E_{\mathcal{M}} \quad \text { and } \quad e_{2}(I)=-E_{I} \cdot E_{I}^{Z} .
$$

We write $I=f \cdot \mathfrak{a}$, where $\mathfrak{a}$ is a zero dimensional ideal and $f$ defines an effective divisor $F$ of $X$. Then we see that

$$
e_{1}(I)=\operatorname{mult}_{Z}(f)
$$

and, since $E_{I}=E_{\mathfrak{a}}+h^{*} F$ and $E_{I}^{Z} \cdot h^{*} F=0$,

$$
e_{2}(I)=-E_{\mathfrak{a}} \cdot E_{I}^{Z}=-E_{\mathfrak{a}}^{2}+E_{\mathfrak{a}} \cdot E_{I}^{X-Z} \geq e(\mathfrak{a}) .
$$

Therefore inequality (13) follows from Theorem 2.2.

The inequality (11) can be strengthened when $\operatorname{dim} X=2$ by choosing carefully the local coordinates before degenerating to monomial ideals. So, in addition to the assumptions of Theorem 2.2, suppose that $X$ is a surface. Then $Z$ is a point of $X$, which we denote by $p$. We write the ideal $I \subset \mathcal{O}_{X, p}$ in the form

$$
I=f \cdot \mathfrak{a},
$$

where $f \in \mathcal{O}_{X, p}$ and $\mathfrak{a}$ is zero dimensional. If $f$ is a unit in $\mathcal{O}_{X, p}$, then we already know that inequality (11) is sharp by Theorem 1.4. Henceforth, we will assume that $\operatorname{mult}_{p}(f)>0$. We consider the divisor $F$ on $X$ defined by $f$. Let $E_{p} \cong \mathbb{P}^{1}$ be the exceptional divisor of the blow-up of $X$ at $p$, and write the projectivized tangent cone $\mathbb{P} C_{p} F \subset E_{p}$ as a divisor $c_{1} P_{1}+\cdots+c_{r} P_{r}$, where $P_{i} \in E_{p}$ are distinct and $c_{i}>0$. Then set

$$
b_{1}=c_{1}+\cdots+c_{r-1} \text { and } b_{2}=c_{r} .
$$

Note that $b_{1}+b_{2}=\operatorname{mult}_{p}(f)$ and $b_{1}=0$ if and only if $r=1$. Let $\mu(I)$ be the inverse of the log canonical threshold of $\left(\mathcal{O}_{X, p}, I\right)$.

Theorem 2.4. With the above notation, we have the following inequality:

$$
4 \mu(I) \operatorname{mult}_{p}(f)-4 b_{1} b_{2}+e(\mathfrak{a}) \geq 4 \mu(I)^{2} .
$$

Proof. Again, it is enough to prove that

$$
4 \mu(I) \operatorname{mult}_{p}(f)-4 b_{1} b_{2}+2 l\left(\mathcal{O}_{X, p} / \mathfrak{a}\right) \geq 4 \mu(I)^{2} .
$$

We proceed as in the proof of Theorem 2.2. We pick a regular system of parameters $x_{1}, x_{2}$ at $p$, such that the tangent direction to $F$ at $p$, corresponding to $P_{r}$, has equation $x_{1}=0$. We take a multiplicative order on the coordinates such that $x_{1}<x_{2}$. 
Using the notation in the proof of Theorem 2.2, we have $J=x_{1}^{b_{1}} x_{2}^{b_{2}}$. $\cdot$ in $(\mathbf{c})$, where $b_{1}$ and $b_{2}$ are the ones defined in (14), and in(c) is a zero dimensional monomial ideal.

Again, we have $\operatorname{mult}_{p}(f)=b_{1}+b_{2}, l\left(\mathcal{O}_{X, p} / \mathfrak{a}\right)=l(R /$ in $(\mathbf{c}))$, and $\mu(I) \leq \mu(J)$. Since $J$ is a monomial ideal, Lemma 2.1 gives

$$
l(R / \mathfrak{a}) \geq 2\left(\mu(J)-b_{1}\right)\left(\mu(J)-b_{2}\right) .
$$

Then, arguing as in the proof of Theorem 2.2, we obtain (16).

Remark 2.5. We remark that, if $r \geq 3$, then there is a certain freedom in choosing $b_{1}, b_{2}$ satisfying (14) by reordering the points $P_{1}, \ldots, P_{r}$. One can check that the strongest inequality is obtained when $c_{r}=$ $\max _{i}\left\{c_{i}\right\}$.

Remark 2.6. In the special case when $\operatorname{Supp}(F)$ has exactly two smooth branches meeting transversally at $p$, Theorem 2.4 implies an earlier result of Corti $\mathrm{Co}$.

We end with a comment about the boundary cases in inequality (15). By suitably adapting the arguments in the proof of Theorem 1.4, we can show the following characterization: under the assumptions of Theorem 2.4, if

$$
4 \mu(I) \operatorname{mult}_{p}(f)-4 b_{1} b_{2}+e(\mathfrak{a})=4 \mu(I)^{2},
$$

then $2 \mu(I) \in \mathbb{N}$ and, if $J$ is as in the proof of Theorem 2.4, then $J$ has integral closure

$$
\bar{J}=x_{1}^{b_{1}} x_{2}^{b_{2}} \cdot \overline{\left(x_{1}^{2 \mu(I)-2 b_{1}}, x_{2}^{2 \mu(I)-2 b_{2}}\right)} .
$$

The example below shows that, under these assumptions, we can not expect to get a characterization for the integral closure of $I$, as we did for zero dimensional ideals in Theorem 1.4. Consider the following ideal in $k\left[x_{1}, x_{2}\right]$ :

$$
I=f \cdot \mathfrak{a}=x_{2}^{2} \cdot\left(x_{1}^{6}, x_{2}^{2}+x_{1}^{2} x_{2}\right) .
$$

Then $\mu(I)=3, \operatorname{mult}_{p}(f)=2, b_{1}=0, b_{2}=2$ and $e(\mathfrak{a})=12$, so equality (17) is satisfied, but $I \not \subset x_{2}^{2} \cdot \overline{\left(x_{1}^{6}, x_{2}^{2}\right)}$.

\section{REFERENCES}

[Co] A. Corti, Singularities of linear systems and 3-fold birational geometry, in Explicit birational geometry of 3-folds, 259-312, Cambridge Univ. Press, Cambridge, 2000.

[CPR] A. Corti, A.V. Pukhlikov and M. Reid, Fano 3-fold hypersurfaces, in Explicit birational geometry of 3-folds, 175-258, Cambridge Univ. Press, Cambridge, 2000 . 
[DEM] T. de Fernex, L. Ein and M. Mustaţă, Bounds for log canonical thresholds, in preparation.

[DK] J.-P. Demailly and J. Kollár, Semi-continuity of complex singularity exponents and Kähler-Einstein metrics on Fano orbifolds, Ann. Sci. École Norm. Sup. (4) 34 (2001), 525-556.

[Ei] D. Eisenbud, Commutative algebra with a view toward algebraic geometry, Grad. Texts in Math. 150, Springer, New York, 1995.

[GG] T. Gaffney and R. Gassler, Segre numbers and hypersurface singularities, J. Algebraic Geom. 8 (1999), 695-736.

[Ho] J. Howald, Multiplier ideals of monomial ideals, Trans. Amer. Math. Soc. 353 (2001), 2665-2671.

[IM] V.A. Iskovskikh and Yu. I. Manin, Three-dimensional quartics and counterexamples to the Lüroth problem, Math. USSR Sbornik 15 (1971), 141166.

[Ko] J. Kollár, Singularities of pairs, in Algebraic Geometry - Santa Cruz 1995, 221-287, Proc. Sympos. Pure Math., 62, Amer. Math. Soc., Providence, RI, 1997.

[Mu M. Mustaţă, Singularities of pairs via jet schemes, J. Amer. Math. Soc. 15 (2002), 599-615.

[Pu1] A.V. Pukhlikov, Birationally rigid Fano hypersurfaces, preprint 2002, math.AG/0201302.

[Pu2] A.V. Pukhlikov, Essentials of the method of maximal singularities, in Explicit birational geometry of 3-folds, 73-100, Cambridge Univ. Press, Cambridge, 2000.

[Pu3] A.V. Pukhlikov, Birational automorphisms of a four-dimensional quintic, Invent. Math. 87 (1987), 303-329.

[Re] D. Rees, $\mathfrak{a}$-transforms of local rings and a theorem on multiplicities of ideals, Proc. Cambridge Philos. Soc. 57 (1961), 8-17.

Department of Mathematics, Statistics and Computer Science, University of Illinois at Chicago, 851 Morgan St., M/C. 249, Chicago, IL 60607-7045,USA

E-mail address: defernex@math.uic.edu

Department of Mathematics, Statistics and Computer Science, University of Illinois at Chicago, 851 Morgan St., M/C. 249, Chicago, IL 60607-7045, USA

E-mail address: ein@math.uic.edu

Isaac Newton Institute for Mathematical Sciences, 20 Clarkson Road, Cambridge, CB3 0EH, England

E-mail address: mirceamustata@yahoo.com 